\title{
Fundamental Function for the Random Walk Simulation under the External Field in One Dimension
}

\author{
Joung-Hahn Yoon and Hyojoon Kim ${ }^{\dagger}, *$ \\ Department of Mathematics, Dong-A University, Busan 604-714, Korea \\ ${ }_{\dagger}^{\dagger}$ Department of Chemistry, Dong-A University, Busan 604-714, Korea. *E-mail: hkim@donga.ac.kr \\ Received April 2, 2014, Accepted June 4, 2014
}

Key Words : Diffusion-reaction, Diffusion-influenced reaction, Monte Carlo simulation, Lattice simulation, External field

Many kinds of diffusion-influenced reactions can be described by the lattice-based random walk Monte Carlo simulations. ${ }^{1-7}$ Recently, we reported the fundamental distribution functions for the lattice-based random walk model in one dimension for the nonreactive and the Smoluchowski boundary conditions. ${ }^{8}$ We also obtained the discrete version of the survival probability for the Smoluchowski boundary condition, ${ }^{9}$ which can reduce to the well-known continuum version result. ${ }^{8}$

The previous results only consider the case where the probability of the movement of the walker is completely random or not affected by other external fields, which include gravitational, magnetic, and electric effects. Since these external field effects on diffusion-reaction systems are ubiquitous in a broad range of chemical and biological systems, ${ }^{9-18}$ it is of use to generalize the previous results to include the external field effects.

\section{Exact Solutions in One Dimension}

Firstly, we consider the random walk model without a reactive trap. Let $P_{N}\left(x, n ; x_{0}\right)$ be the non-reactive probability that the walker is observed at $x$ after $n$ steps with the starting position $x_{0}$ on the one-dimensional lattice. Suppose that jumps to the left and to the right occur with probability $p$ and $q=1-p$, respectively. Then, the probability that the walker moved $k$ times to the left and $n-k$ times to the right is given by the binomial distribution

$$
P_{N}\left(x, n ; x_{0}\right)={ }_{n} C_{k} p^{k} q^{n-k}
$$

where ${ }_{n} C_{k}=n ! /\{k !(n-k) !\}$ and $k=\left(x_{0}+n-x\right) / 2$ should be an integer satisfying $-n+x_{0} \leq x \leq n+x_{0}$. Unless $x_{0}+n-x$ is a non-negative even number, $P_{N}$ is zero.

The random walk model with a single static perfect trap can be considered. This model corresponds to diffusionreaction systems with the Smoluchowski or the absorbing boundary condition. In other words, the random walker cannot escape once trapped. Let $P_{S M}\left(x, n ; x_{0}\right)$ be the probability that the walker is observed at $x$ after $n$ steps with the starting position $x_{0}$ for the Smoluchowski boundary condition. Without loss of generality, we can assume that the trap is located at the origin and the starting position is $x_{0}>0$. Then, we find

$P_{S M}\left(x, n ; x_{0}\right)=\left\{\begin{array}{lr}{ }_{n} C_{k} p^{k} q^{n-k}-{ }_{n} C_{k-x_{0}} p^{k} q^{n-k} & 0<x \leq n-x_{0} \\ { }_{n} C_{k} p^{k} q^{n-k} & n-x_{0}<x \leq n+x_{0}\end{array}\right.$

Note that $n+x_{0}$ is the maximum distance after $n$ steps. Since ${ }_{n} C_{k-x_{0}} p^{k} q^{n-k}=(p / q){ }_{n}^{x_{0}} C_{k-x_{0}} p^{k-x_{0}} q^{n-k+x_{0}}=(p / q){ }^{x_{0}} P_{N}\left(x, n ;-x_{0}\right)$, we have the following relation,

$$
\begin{aligned}
& P_{S M}\left(x, n ; x_{0}\right) \\
& \quad=\left\{\begin{array}{lr}
P_{N}\left(x, n ; x_{0}\right)-\left(\frac{p}{q}\right)^{x_{0}} P_{N}\left(x, n ;-x_{0}\right) & 0<x \leq n-x_{0} \\
P_{N}\left(x, n ; x_{0}\right) & n-x_{0}<x \leq n+x_{0}
\end{array}\right.
\end{aligned}
$$

This relation between $P_{S M}$ and $P_{N}$ generalizes the previous result with equal jumping probabilities. Note that $P_{S M}$ is zero at every other $x$ like $P_{N}$.

The survival probability $S_{S M}\left(n ; x_{0}\right)$ can be obtained by summing $P_{S M}\left(x, n ; x_{0}\right)$ of Eq. (3) over all possible $x$ as

$$
\begin{aligned}
& S_{S M}\left(n ; x_{0}\right)=\sum_{x=1}^{n+x_{0}} P_{S M}\left(x, n ; x_{0}\right) \\
& \quad=\sum_{x=1}^{n+x_{0}} P_{N}\left(x, n ; x_{0}\right)-\left(\frac{p}{q}\right)^{x_{0}} \sum_{x=1}^{n-x_{0}} P_{N}\left(x, n ;-x_{0}\right) .
\end{aligned}
$$

Note that the last term comes from the summation only over $1 \leq x \leq n-x_{0}$. Since $P_{N}\left(x, n ;-x_{0}\right)=P_{N}\left(x+2 x_{0}, n ; x_{0}\right)$, Eq. (4) can be rearranged as

$$
S_{S M}\left(n ; x_{0}\right)=\sum_{x=1}^{n+x_{0}} P_{N}\left(x, n ; x_{0}\right)-\left(\frac{p}{q}\right)^{x_{0}} \sum_{x=1+2 x_{0}}^{n+x_{0}} P_{N}\left(x, n ; x_{0}\right) \text {. }
$$

Because of the property of $P_{N}, S_{S M}\left(n+1 ; x_{0}\right)=S_{S M}\left(n ; x_{0}\right)$ holds when $x_{0}+n$ is even.

\section{Relation to the Solutions in Diffusion-influenced Reactions}

As $n$ increases, the following well-known de MoivreLaplace theorem ${ }^{19}$ can be used

$$
{ }_{n} C_{k} p^{k} q^{n-k} \cong \frac{1}{\sqrt{2 \pi n p q}} \exp \left(-\frac{(k-n p)^{2}}{2 n p q}\right),
$$


for $k$ in the neighborhood of $n p$. This theorem means that in the large $n$ limit or in the long time limit, the discrete binomial distribution $B(n, p)$ can be approximated by the normal distribution function $N(n p, n p q)$ with the mean value $n p$ and the variance $n p q$ especially in the vicinity of $k=n p$. Then, we obtain

$$
P_{N}\left(x, n ; x_{0}\right) \cong \frac{1}{\sqrt{8 \pi n p q}} \exp \left[-\frac{\left\{x-x_{0}+n(p-q)\right\}^{2}}{8 n p q}\right] .
$$

Note that this approximation is better when $x-x_{0}=n(q-p)$, namely, Eq. (6) is better when $p=q=1 / 2$ around $x=x_{0}$. Since $P_{N}$ is zero at every other $x$ in the discrete version, we have multiplied the factor $1 / 2$. We can relate the parameters in the discrete version and those in the continuum version. When $D$ is the diffusion constant, $t$ is the time, and $a$ is the dimensionless external field strength, we have $n=2 D t$ and

$$
p=\frac{e^{a}}{e^{a}+e^{-a}}, q=\frac{e^{-a}}{e^{a}+e^{-a}}
$$

Simple computation leads to $p q=1 /(2 \cosh a)^{2}, p / q=e^{2 a}$, and $p-q=\tanh a$. From Eq. (7), we have

$$
\begin{aligned}
& P_{N}\left(x, n ; x_{0}\right) \cong \frac{\cosh a}{\sqrt{4 \pi D t}} \\
& \quad \exp \left[-\frac{\left(x-x_{0}\right)^{2} \cosh ^{2} a}{4 D t}-\frac{\left(x-x_{0}\right) \sinh 2 a}{2}-D t \sinh ^{2} a\right] .
\end{aligned}
$$

In the small field strength limit or $a \approx 0, \cosh a \approx 1$ and $\sinh a \approx a$, then we can reduce Eq. (9) to the well-known expression $^{11}$

$$
\lim _{a \rightarrow 0} P_{N}\left(x, n ; x_{0}\right) \cong \frac{e^{-a\left(x-x_{0}+a D t\right)}}{\sqrt{4 \pi D t}} \exp \left[-\frac{\left(x-x_{0}\right)^{2}}{4 D t}\right] .
$$

When we compare the results from Eqs. (9) and (10) with those from Eq. (1), we find that Eq. (10) usually produces results with smaller deviations than Eq. (9). This can be understood by the fact that Eq. (6) better describes the situation with $a \approx 0$. Therefore, the following approximation is found to be better in our model,

$$
\lim _{a \rightarrow 0} C_{k} p^{k} q^{n-k} \cong \sqrt{\frac{1}{2 \pi n}} \exp \left(-\frac{\left(x-x_{0}+n a\right)^{2}}{2 n}\right),
$$

with $a=\tanh ^{-1}(2 p-1)$. Then, for the $P_{S M}$ function of Eq. (2), we can have the known expression of ${ }^{11}$

$$
\begin{aligned}
& \lim _{a \rightarrow 0} P_{S M}\left(x, n ; x_{0}\right) \cong \frac{e^{-a\left(x-x_{0}+a D t\right)}}{\sqrt{4 \pi D t}} \\
& {\left[\exp \left\{-\frac{\left(x-x_{0}\right)^{2}}{4 D t}\right\}-\exp \left\{-\frac{\left(x+x_{0}\right)^{2}}{4 D t}\right\} .\right.}
\end{aligned}
$$

The survival probability $S_{S M}\left(n ; x_{0}\right)$ of Eq. (5) can be approximated by the following integral:

$$
\begin{aligned}
\lim _{a \rightarrow 0} S_{S M}\left(n ; x_{0}\right) \cong & \sqrt{\frac{1}{2 \pi n}}\left(\int_{0}^{\infty} \exp \left[-\frac{\left\{x-x_{0}+n a\right\}^{2}}{2 n}\right] d x\right. \\
& \left.-e^{2 a x_{0}} \int_{2 x_{0}}^{\infty} \exp \left[-\frac{\left\{x-x_{0}+n a\right\}^{2}}{2 n}\right] d x\right) \\
= & \int_{-x_{0}+n a}^{\infty} \sqrt{\frac{1}{\pi}} \exp \left[-u^{2}\right] d u-e^{2 a x_{0}} \int_{\frac{x_{0}+n a}{\sqrt{2 n}}}^{\infty} \sqrt{\frac{1}{\pi}} \exp \left[-u^{2}\right] d u, \\
= & \frac{1}{2} \operatorname{erfc}\left(\frac{-x_{0}+n a}{\sqrt{2 n}}\right)-e^{2 a x_{0}} \frac{1}{2} \operatorname{erfc}\left(\frac{x_{0}+n a}{\sqrt{2 n}}\right),
\end{aligned}
$$

where $\operatorname{erfc}(x)$ is the complementary error function. The second equality comes from the substitution of $(2 x-n)^{2}=$ $2 n u^{2}$. If $n=2 D t$, this equation is again the same as the wellknown survival probability with the Smoluchowski boundary condition $^{11}$

$$
\lim _{\substack{n \rightarrow \infty \\ a \rightarrow 0}} S_{S M}\left(n ; x_{0}\right) \cong 1-\frac{1}{2} \operatorname{erfc}\left(\frac{x_{0}-2 a D t}{\sqrt{4 D t}}\right)-\frac{e^{2 a x_{0}}}{2} \operatorname{erfc}\left(\frac{x_{0}+2 a D t}{\sqrt{4 D t}}\right) \text {. }
$$

Therefore, the discrete exact solutions can be reduced to the known corresponding solutions in diffusion-influenced reactions only in the small field strength limit.

\section{Monte Carlo Simulation Results}

To confirm the present results, we perform the Monte

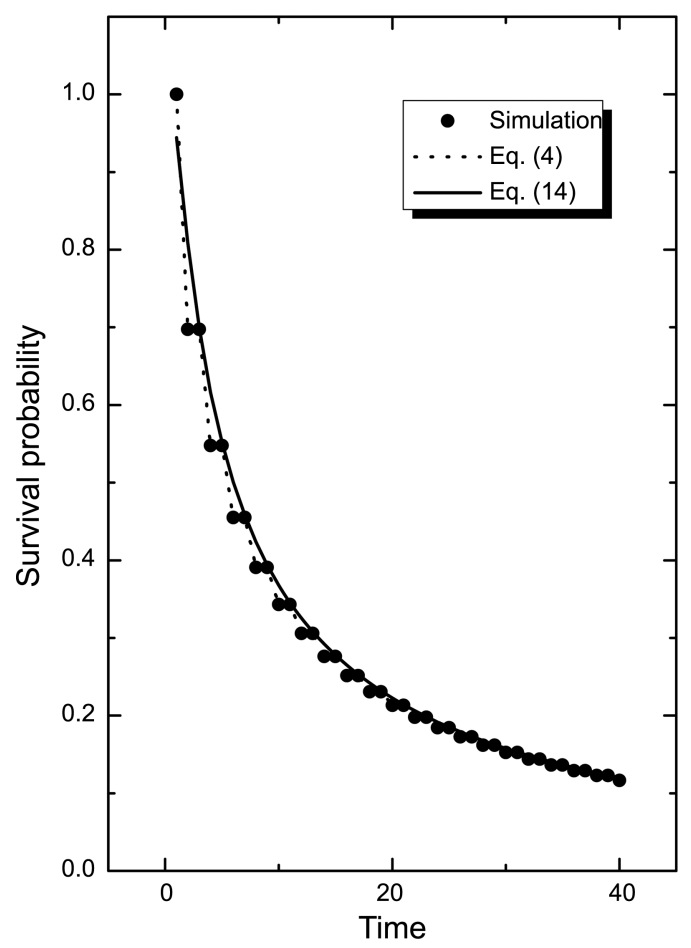

Figure 1. The time-dependence of the survival probability function for $x_{0}=2$ and $p=0.55$. Closed circles are from the simulation results, which are in perfect agreement with those from Eq. (4) in the dotted line. The approximated results from Eq. (14) are plotted in the solid line. 


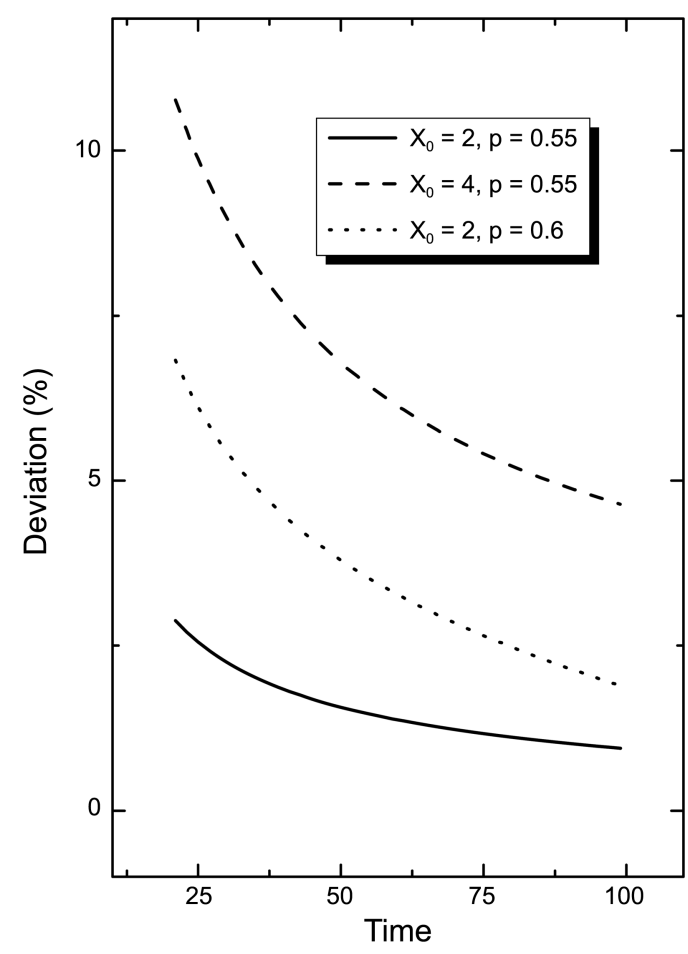

Figure 2. The time-dependent deviations of the approximated survival probability function of Eq. (14) from its discrete version of Eq. (4) for three given conditions.

Carlo simulations of the latticed-based random walk model. ${ }^{2-6}$ After a particle is initially implanted at $x_{0}$, it starts moving in random directions (one of two directions in this case) until it reaches the origin where the trap exists. Under the Smoluchowski boundary condition, the reaction always occurs when the particle moves to the trap. Therefore, when trapped, we do not have to follow the trajectory and start a new trajectory. Ten million trajectories are averaged to obtain the converged numerical results.

In Figure 1, we plot the survival probabilities for $x_{0}=2$ and $p=0.55$ in unit-dimensionless variables. ${ }^{20}$ In one dimensional lattice, $D=1 / 2$. One can confirm that Eq. (4) perfectly reproduce the simulation results. Note that $a$ can be obtained by $a=\tanh ^{-1}(2 p-1)$ from Eq. (8). Therefore, we can obtain the exact results more efficiently from Eq. (4) than from simulations which have an inevitable statistical noise. It should be noted that $S_{S M}\left(n+1 ; x_{0}\right)=S_{S M}\left(n ; x_{0}\right)$ holds. One can also see that the discrete [Eq. (4)] and the continuum results [Eq. (14)] are in excellent agreement with each other. The difference between two is quantified in Figure 2, where the deviations of Eq. (14) from Eq. (4) are compared for three conditions. For the condition of Figure 1, the deviations reduce from approximate $3 \%$ to $1 \%$ as time goes by. For the larger $x_{0}$ and the larger $p$, the deviations increase as expected from the approximations of Eq. (11).

In summary, the fundamental distribution functions for the lattice-based random walk model in one dimension under the influence of the external field effects are found for the nonreactive and the Smoluchowski boundary conditions. The discrete survival probability function for the Smoluchowski boundary condition is also found. Thus, the previous results ${ }^{8}$ are generalized to include the external field effects. The numerical simulation results can be replaced by these superior analytic functions. These discrete functions are confirmed to reduce to their corresponding continuum version results only in the small field strength limit. Therefore, we have to be careful to simulate the system affected by the external field. The field effects between neighboring points should be small enough usually by decreasing the lattice constant, ${ }^{20}$ which increases the computational cost. One important merit of this work is that we can find an optimized lattice constant by quantifying the deviations caused by the field effects.

Acknowledgments. This work was supported by research funds from Dong-A University.

\section{References}

1. ben-Avraham, D.; Havlin, S. Diffusion and Reactions in Fractals and Disordered Systems; Cambridge University Press: Cambridge, 2000.

2. Kim, H.; Shin, S.; Lee, S.; Shin, K. J. J. Chem. Phys. 1996, 105, 7705 .

3. Kim, H.; Shin, S.; Shin, K. J. J. Chem. Phys. 1998, 108, 5861.

4. Kim, H.; Shin, S.; Shin, K. J. Chem. Phys. Lett. 1998, 291, 341.

5. Kim, H.; Shin, K. J. Phys. Rev. E 2000, 61, 3426.

6. Kim, H. Chem. Phys. Lett. 2010, 484, 358.

7. Montroll, E. W.; Weiss, G. H. J. Math. Phys. 1965, 6, 167.

8. Yoon, J. H.; Kim, H. Bull. Korean Chem. Soc. 2011, 32, 3521.

9. Smoluchowski, M. Z. Phys. Chem. (Leipzig) 1917, 92, 129.

10. Chandrasekhar, S. Rev. Mod. Phys. 1943, 15, 1.

11. Carslaw, H. S.; Jaeger, J. C. Conduction of Heat in Solids, 2nd ed.; Oxford University Press: New York, 1986.

12. Kim, H.; Shin, K. J.; Agmon, N. J. Chem. Phys. 2001, 114, 3905.

13. Kim, H.; Shin, K. J. J. Chem. Phys. 2004, 120, 9142.

14. Park, S.; Shin, K. J. Chem. Asian J. 2006, 1, 216.

15. Park, S.; Shin, K. J. J. Phys. Chem. B 2008, 112, 6241.

16. Reigh, S. Y.; Shin, K. J.; Tachiya, M. J. Chem. Phys. 2008, 129, 234501.

17. Reigh, S. Y.; Shin, K. J.; Kim, H. J. Chem. Phys. 2010, 132, 164112.

18. Reigh, S. Y.; Kim, H. Bull. Korean Chem. Soc. 2012, 33, 1015.

19. Feller, W. An Introduction to Probability Theory and Its Applications, 3rd ed.; Wiley: New York, 1968; Vol. 1

20. Kim, T.; Kim, H. Bull. Korean Chem. Soc. 2014, 35, 1209. 